\title{
Correction to: Sucrose isomers as alternative sweeteners: properties, production, and applications
}

\author{
Yuqing Tian ${ }^{1} \cdot$ Yu Deng ${ }^{2,3} \cdot$ Wenli Zhang ${ }^{1,4}(D)$ Wanmeng Mu ${ }^{1,4}$ \\ Published online: 14 November 2019 \\ (C) Springer-Verlag GmbH Germany, part of Springer Nature 2019
}

\section{Correction to: Applied Microbiology and Biotechnology https://doi.org/10.1007/s00253-019-10132-6}

There is an error in the original publication of this paper. Figures 1 and 2 have been interchanged. The corrected version of these figures is shown below:

The online version of the original article can be found at https://doi.org/ 10.1007/s00253-019-10132-6

\footnotetext{
Yu Deng

dengyu@jiangnan.edu.cn

$\triangle$ Wenli Zhang

wenlizhang@jiangnan.edu.cn

1 State Key Laboratory of Food Science and Technology, Jiangnan University, Wuxi 214122, Jiangsu, China

2 National Engineering Laboratory for Cereal Fermentation Technology, Jiangnan University, Wuxi 214122, Jiangsu, China

3 School of Biotechnology, Jiangnan University, Wuxi 214122, Jiangsu, China

4 International Joint Laboratory on Food Safety, Jiangnan University, Wuxi 214122, Jiangsu, China
} 


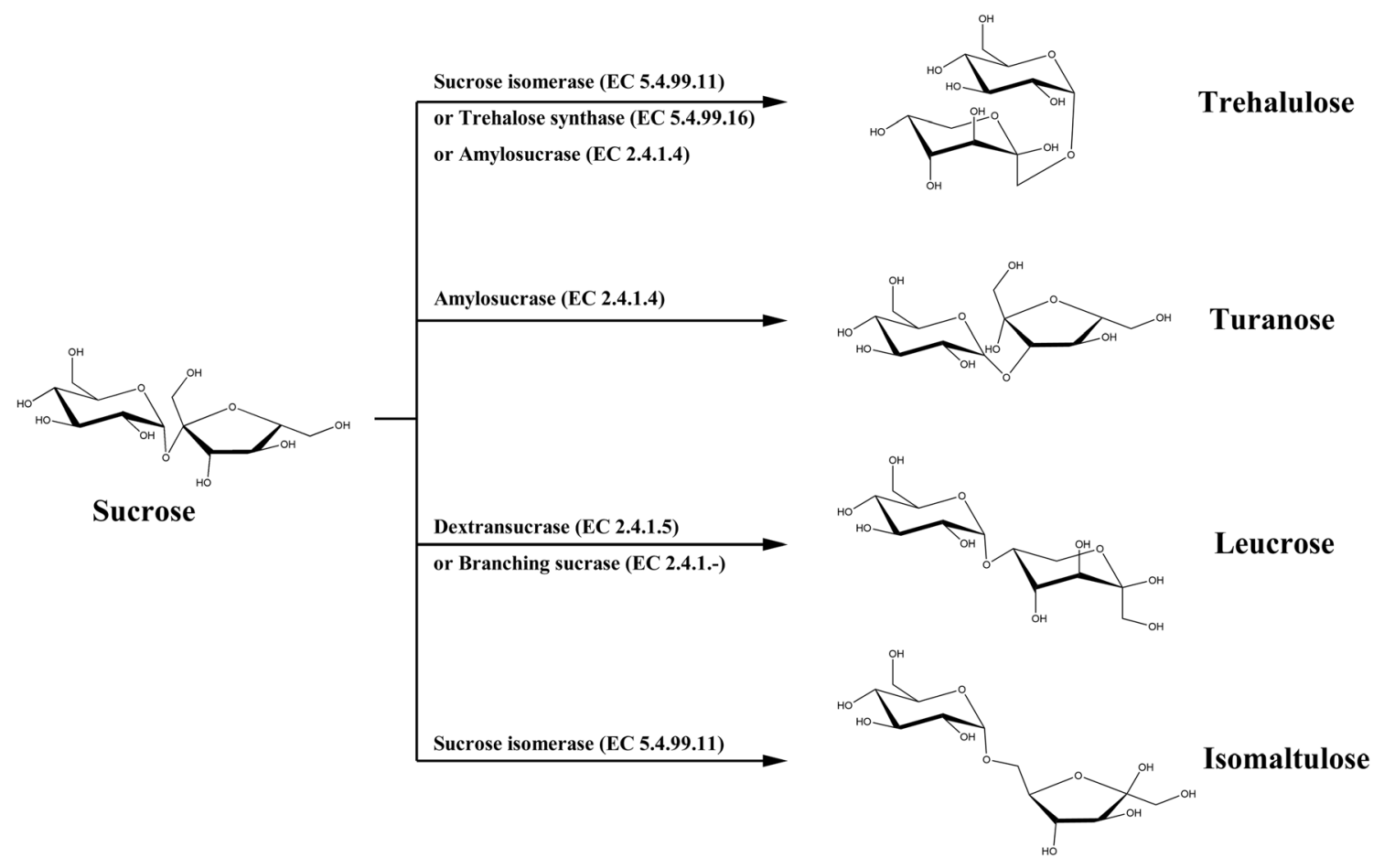

Fig. 1 An overview of the enzymatic synthesis of four sucrose isomers from sucrose 


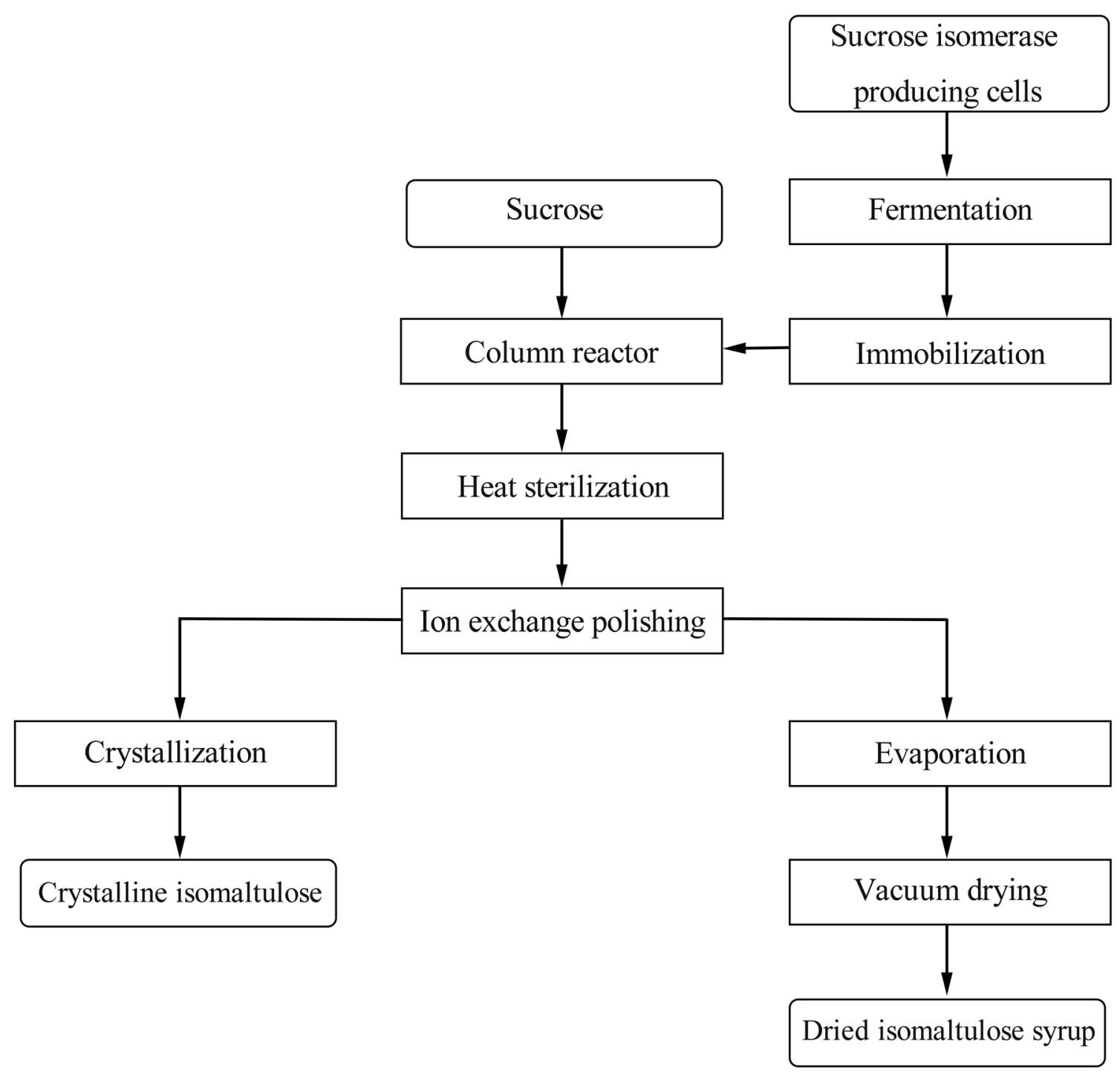

Fig. 2 A flowchart of the typical process of isomaltulose production on a large scale

Publisher's note Springer Nature remains neutral with regard to jurisdictional claims in published maps and institutional affiliations. 